\title{
The Andago for overground gait training in patients with gait disorders after stroke - results from a usability study
}

\author{
Detlef Marks ${ }^{1 *}$, Raoul Schweinfurther ${ }^{1}$, Alexander Dewor ${ }^{1}$, Tommy Huster ${ }^{1}$, Liliana P Paredes ${ }^{1}$, Daniel Zutter ${ }^{1}$ and Jens C Möller ${ }^{1,2}$ \\ ${ }^{1}$ Rehaklinik Zihlschlacht, Centre for Neurological Rehabilitation, Zihlschlacht, Switzerland \\ ${ }^{2}$ Department of Neurology, Philipps University, Marburg, Germany
}

\begin{abstract}
Background: Mobility training is an important goal of rehabilitation. The Andago is a recently developed device allowing body weight supported overground mobility training.

Objective: The aim of this study was to evaluate the usability of the prototype Andago V2.0 and its acceptance by patients and therapists.

Methods: The trial was designed as an exploratory study without control group. Patients were recruited from inpatients training on the Lokomat. The Andago was first tested in a training session to familiarize the participants with the device. The following therapy session included a course predefined by our infrastructure. Therapists/ patients rated handling, usability and satisfaction.

Results: Fifteen patients were eligible and gave informed consent. Two patients were excluded due to protocol violation. Patient characteristics: age 58, NIHSS 8.3, FAC 1.3 (all mean), male 8, days since stroke onset 65 (median). All sessions were performed safely. No Adverse Events (AE) or Adverse Device Effects (ADE) occurred. Patients reported feeling safe. Therapists had to steer accurately, e.g., while passing through doors or turning. Hemianopia and/or neglect frequently led to interruptions of the Andago sessions.
\end{abstract}

Conclusions: Most of the patients appreciated the device. This study shows, that Andago can be used safely under therapeutic supervision for overground gait training in stroke patients presenting moderate to severe gait disorders.

Trial registration: The study was registered under ClinicalTrials.gov (Identifier: NCT02735460) and in the Swiss National Clinical Trials Portal.

\begin{abstract}
Abbreviations: AE: Adverse Events; ADE: Adverse Device Effects; BWS: Body weight support; $\mathrm{cm}$ : centimetre; FAC: Functional Ambulation Categories; kg: kilogram; ICD: International Classification of Diseases; m: meter; MAM: Manual mode; PFM: Patient-following mode; NIHSS: National Institute of Health Stroke Scale; SAE: Serious Adverse Events; SLM: Straight-line mode; 10-MWT: Timed 10-meter walk test.
\end{abstract}

\section{Background}

Gait disorder is a prevalent symptom in patients with neurological disorders and one of the most frequently targeted symptoms in neurorehabilitation. A variety of neurological gait disorders such as paretic, spastic, hypokinetic or ataxic gait can be distinguished [1]. The gait pattern in patients after stroke is characterized by asymmetry, poor motor control, impaired balance and reduced weight bearing on the paretic limb [2]. Smooth and symmetrical forward progression during walking is impaired. Mass movements on the paretic side require compensatory adjustments of the pelvis and nonparetic side. Gait disorders after stroke are basically not responsive to pharmacological therapy. Therefore, neurorehabilitation plays a prominent role in the treatment of hemiparetic gait in stroke patients. In addition to conventional physiotherapy, electromechanically assisted therapies have gradually gained in importance in the rehabilitation of these patients promising improved outcome [3]. A recent review by the Cochrane Collaboration provided evidence that the use of electromechanically assisted body weight supported gait training devices in combination with physiotherapy increases the chance of regaining independent walking ability for patients after stroke [4].
Despite the efficacy of electromechanically assisted gait training, overground gait training is needed for several reasons to transfer the acquired skills to activities of daily living in patients making the transition from non-walkers to walkers [5]. First, the requirements for propulsion and balance control may differ between robot-assisted and overground gait. Second, walking speed is not self-selected when walking on a treadmill or an electromechanically assisted device [6]. Accordingly, during overground training stroke patients have to walk more independently, generate steps without support and maintain balance with the help of a therapist. Apart from that, it has been shown that more frequent therapy has a favourable effect on activities of daily living and gait speed [7]. The need of overground walking practice is traditionally met through the assistance of therapists and the use of walking aids such as canes or walkers. Systems using body weight support (BWS) for "indoor walking" could therefore be useful for more frequent mobility training. Hence, the Andago may help bridge the

${ }^{\star}$ Correspondence to: Detlef Marks, Rehaklinik Zihlschlacht Hauptstr. 2-4 CH-8588 Zihlschlacht, Switzerland, Tel: +41-71-424-3751; E-mail: d.marks@ rehaklinik-zihlschlacht.ch

Key words: Andago, stroke, overground gait training, mobility, electromechanical gait trainer, body weight support

Received: November 01, 2019; Accepted: November 21, 2019; Published: November 28, 2019 
gap between robot-assisted therapy and conventional overground gait training in stroke patients.

\section{Objective}

In this paper, we introduce the Andago V2.0, the prototype of a medical device for dynamic body weight supported overground gait and balance training of patients with (neurological) gait disorders such as stroke which will be referred to as "Andago". A CE-certified commercial version of the Andago is now available (https://www.hocoma.com/us/ solutions/andago/).

Andago consists of a frame on wheels with an integrated BWS system. It senses movements of the trunk of the patient through sensors in the carriers and automatically follows the patient allowing gait training without being confined to a single training room. Andago is composed by two BWS modules, two lifts and a mobile platform module and controlled via a handheld by the therapist allowing different training modes:

- Patient-following mode (PFM): Andago senses the patient movements and the mobile platform behaves according to these movements, following the patient in a transparent way in the forward and backward directions and right and left turns.

- Straight-line mode (SLM): Andago follows the patient only in the forward/backward direction and rejects any input for turning.

- Manual mode (MAM): The therapist controls the movement of the Andago via joystick in the upper part of the handheld (forward/ backward movements and turns are also possible).

Accordingly, the aim of the present study was to address for the first time the usability and acceptance of the Andago by patients and therapists, focusing on its performance (e.g., training modes, device settings or training conditions) during inpatient neurorehabilitation.

\section{Materials and methods}

\section{Design}

We conducted an exploratory, open-label, uncontrolled usability study to analyse the practicability and acceptance of the Andago. The goal was to analyse usability outcomes like setup and dismounting times, patient and therapist satisfaction and the occurrence of $\mathrm{AE}$ in stroke patients under real-life conditions in the setting of a neurorehabilitation clinic. The study protocol was approved by the local Ethics Committee (Ethikkommission des Kanton Thurgau, KEKTGOV 2015/29) and Swissmedic (2015-MD-0033). Informed consent was obtained from each patient. Besides, the study was subject to a monitoring by Clinical Trials Centre Zürich (http://www.ctc-zkf.usz.ch).

\section{Participants}

Patients with gait disorder after ischemic or haemorrhagic stroke who were admitted to Rehaklinik Zihlschlacht for neurorehabilitation were recruited for this study. We consecutively included all inpatients who already trained on the Lokomat due to a clinically relevant gait disorder $[4,8]$. The Lokomat is an exoskeleton that guides the limbs through the gait cycle, making it possible to elicit a kinematically normal gait pattern in patients who are incapable of independent stepping [9]. All patients fulfilled the following inclusion criteria:

- Primary diagnosis of cerebral infarction (ICD-10: I63.0-I63.9) or intracerebral haemorrhage (ICD-10: I61.0-I61.9) independent of its location, age or cause
- Unable to walk without physical assistance: Functional Ambulation Categories $(\mathrm{FAC}) \leq 2[10]$

- Age: $>18$ years

- Weight: $<135 \mathrm{~kg}$

- Height: $<200 \mathrm{~cm}$

Exclusion criteria for Andago use were similar to those of the Lokomat and included:

- Inability to understand the informed consent or to follow the procedures of the study, e.g., due to language problems, psychiatric disorders, cognitive impairment or aphasia (item 9 of the National Institute of Health Stroke Scale (NIHSS) $\geq 2)[11,12]$

- Bone fragility, unstable arthroplasty, uncontrolled knee or ankle instability, relevant joint contractures, relevant differences in leg length

- Lack of head control

- Skin lesions (including pressure sores or enteric stomata) in areas of contact with harness support or lower extremity loading (feet)

- Recent history or significant risk of seizures

- Relevant sensory impairment in the lower limbs and trunk, especially with reduced pain sensation

- Mechanical ventilation

- Long-term infusions (e.g., baclofen pump, other intrathecal pumps)

- Relevant cardiovascular conditions, e.g., cardiac insufficiency and thoracotomy, uncontrolled orthostatic hypotension or other circulatory problems, vascular disorders of the lower limbs

- Any medical condition preventing active rehabilitation and/or the use of the Andago (e.g., respiratory disease, pregnancy, orthopaedic conditions, infections or inflammatory disorders, osteomyelitis)

- Participation in another interventional study within the 30 days preceding and during the present study

\section{Intervention}

During the screening visit the demographic data and comorbidities according to medical history were assessed and a physical exam was performed. The type of stroke according to the current version of the International Statistical Classification of Diseases and Related Health Problems (ICD-10), the TOAST classification in case of ischemic stroke, stroke severity according to the NIHSS, gait disorder according to the Functional Ambulation Categories (FAC) and the location and time since stroke were documented [10-12]. The presence of hemianopia and neglect were defined by a score $\geq 2$ on item 3 and a score $\geq 1$ on item 11 of the NIHSS [13], respectively.

All participants performed a training and a therapy session $(1 \mathrm{~h}$ duration each) with the Andago within 3 weeks from study inclusion. Two therapists with longstanding experience in robot-assisted therapies ( $>10$ years) supervised the session. Usability and acceptance were assessed by the patient and the therapist at each session. AE and other safety-related events were recorded by the therapist. A 10-meter walking test (10-MWT) was performed before and during the therapy session. A focus of both sessions was to measure the times spent preparing the patient, setting up the Andago and dismounting the patient from the device afterwards. This included eleven components (positioning 
Andago, choosing harness, mounting harness, attaching to Andago, lifting patient, setup BWS, setup fall limit, setup speed level, setup turning offset, lower patient, dismount harness). We also evaluated the overall time needed for these steps under real-life conditions: overall time before 1st step (components 1-9) and overall time after last step (components 10-11). For patient preparation in both sessions, the therapist brought Andago to the appropriate starting position by either moving the patient on a wheelchair towards the device from the back of Andago or moving Andago backwards towards the sitting patient. The harness was fitted in standing position with help of one therapist. Subsequently, the patient lift was moved down to the point where the harness loops could be attached to the lift straps. Then, the patient was lifted by using the handheld control to a suitable standing position. Subsequently, the BWS, the fall limit and the maximum speed were defined. During the session, the therapist could adapt the parameters. The turning offset could be used in case of gait deviation. Symmetrical BWS was routinely used during the training and therapy sessions. At the end of the session, the therapist stopped Andago with the handheld control and dismounted the patient from the device in a standing position. Finally, the patient was moved down on the wheelchair.

During both sessions, two people were always present. The therapist was in charge of the patient with the Andago and the investigator recorded the study data.

The training session took place within a mean of 4 days after the screening visit. In this session, the therapist always started with the PFM for proper setup and customization. Afterwards he changed the training mode to the SLM and MAM, respectively, and adjusted the settings as necessary. The therapist chose the settings individually at his discretion. The participants were asked to walk back and forth along a straight line of $10 \mathrm{~m}$. The $180^{\circ}$ turns were performed in the MAM, if necessary. This session was performed in a large gym $(12.5 \times 22.5 \mathrm{~m})$. Each training mode was used for 5 minutes, unless the patient experienced strong fatigue. The investigator recorded the preparation and dismounting times, the achieved training times and distances, the parameter settings and any near-falls, collisions, interventions, emergency stops and error messages according to the Andago manual. In addition, the therapist and the patient filled out questionnaires.

The therapy session was performed within 2 weeks from the training session. During this session the patient performed a standardized course predefined by the infrastructure of our clinic, which reflects the usability and acceptance of Andago under real-use conditions:

First, the patient had to pass through a door (min. width $90 \mathrm{~cm}$ ), turn $90^{\circ}$ to the left, follow a 10 - $\mathrm{m}$ straight stretch, turn $90^{\circ}$ to the right, pass through a sliding door, follow a $20-\mathrm{m}$ straight stretch, turn around $\left(180^{\circ}\right.$ turn) and return to the starting point following the same path. The maximum allowed walking time for the therapy session was 20 minutes. The session was stopped if the patient experienced strong fatigue. The same documentation was performed as for the training session. Walking aids such as ankle-foot orthoses were not allowed during the sessions.

\section{Assessments}

\section{Primary outcome}

Primary outcomes of this study were the usability and acceptance of the Andago. Usability was assessed by the time spent on preparation and release of the participant, the achieved training times and/or distances, the used parameter settings, the number of near-falls, collisions and emergency stops. Additionally, the number and type of interventions by the therapist and error messages were recorded. Acceptance was assessed by self-developed satisfaction questionnaires filled out by the patients and the two therapists due to missing published and validated German questionnaires matching our criteria.

\section{Secondary outcomes}

The secondary outcome was the performance of the patient in the 10-MWT [14] with and without the Andago. In the 10-MWT the participant was asked to walk $10 \mathrm{~m}$ without physical assistance at comfortable walking speed. Canes and walking frames were allowed as assistive devices for walking without Andago.

\section{Safety outcomes}

Near-falls and collisions as recorded by the study personnel, interventions by the therapists and emergency stops were assessed for the analysis of the primary outcome of this usability study. Error messages listed in the Andago prototype user manual were recorded. Due to reduced bumper sensitivity of the Andago prototype available for this study, error messages due to collisions were not taken into consideration. For each session the patient was screened for the occurrence of $\mathrm{AE}$ or possibly Serious Adverse Events (SAE). Furthermore, all device deficiencies or ADE were documented. For the analysis of the influence of hemianopia and/or neglect on the use of Andago, all collisions, interventions by the therapists to avoid impending collisions, verbal instructions during the therapy session and physical contacts with the device were documented.

\section{Statistics}

A number of 15 participants allowing for a considerable number of drop-outs and an estimated final sample size of at least ten participants were planned based on our clinical experience with other medical devices used in the setting of our neurorehabilitation clinic. IBM SPSS Statistics 23.0 was used for creating the raw data base (IBM Corp., Armonk, NY). Analysis was conducted in R and figures were produced using the package ggplot2 (R Foundation for Statistical Computing, Vienna, Austria).

For the presentation of the questionnaire data in a table, items were dichotomized if necessary. The Kruskal-Wallis test was used to compare the reached distances at both sessions depending on the FAC. We applied the $\mathrm{t}$-test to compare the reached distances and devices for walking without Andago and the Wilcoxon rank-sum test to analyse the frequency of events in patients with and without hemianopia/neglect, respectively. For analysis of the $10-\mathrm{MWT}$ we used a paired t-test. The $\mathrm{p}$-value for statistically significant differences was defined as 0.05 .

\section{Results}

\section{Study population}

15 patients were included into this study. Two patients had to be excluded due to a protocol violation during the training session. The remaining 13 patients were used for the analysis of this usability study. The demographic and clinical data of these patients are shown in table 1.

\section{Usability and acceptance}

On average $437 \pm 138 \mathrm{sec}$ was needed for the setup time before the first walking step and $96 \pm 31 \mathrm{sec}$ for dismounting the patient from Andago. Descriptively, we did not observe any remarkable difference in these times with respect to the FAC of the participant or the therapist performing the training during both sessions (Figure 1). 
Marks D (2019) The Andago for overground gait training in patients with gait disorders after stroke - results from a usability study

Table 1. Demographic and clinical data of the study population $(n=13)$

\begin{tabular}{|c|c|c|c|c|c|c|c|c|}
\hline ID & Gender (F/M) & Age (years) & Days since onset & Affected side & Type of stroke & FAC & NIHSS & $\begin{array}{c}\text { Hemianopia and } / \\
\text { or neglect }\end{array}$ \\
\hline 1 & $\mathrm{~F}$ & 57 & 63 & Right & Infarction & 2 & 8 & Yes \\
\hline 2 & M & 64 & 53 & Right & Hemorrhage & 0 & 10 & Yes \\
\hline 3 & M & 61 & 3841 & Right & Infarction & 2 & 6 & Yes \\
\hline 4 & M & 40 & 138 & Right & Infarction & 2 & 11 & Yes \\
\hline 5 & M & 70 & 42 & Right & Infarction & 0 & 10 & Yes \\
\hline 6 & M & 33 & 1299 & Left & Hemorrhage & 1 & 8 & No \\
\hline 7 & $\mathrm{M}$ & 75 & 151 & Right & Hemorrhage & 1 & 9 & No \\
\hline 8 & $\mathrm{~F}$ & 57 & 54 & Right & Hemorrhage & 2 & 7 & Yes \\
\hline 9 & M & 78 & 26 & Right & Infarction & 2 & 4 & Yes \\
\hline 10 & $\mathrm{~F}$ & 30 & 245 & Right & Hemorrhage & 0 & 10 & No \\
\hline 11 & $\mathrm{~F}$ & 40 & 65 & Left & Hemorrhage & 0 & 12 & No \\
\hline 12 & M & 65 & 10 & Right & Infarction & 2 & 3 & No \\
\hline 13 & $\mathrm{~F}$ & 79 & 67 & Right & Infarction & 1 & 10 & Yes \\
\hline $\mathrm{N}$ & $\begin{array}{l}8 \mathrm{M} \\
5 \mathrm{~F}\end{array}$ & & & $\begin{array}{l}11 \text { Right } \\
2 \text { Left }\end{array}$ & $\begin{array}{l}7 \text { Infarctions } \\
6 \text { Haemorrhage }\end{array}$ & & & $\begin{array}{l}8 \mathrm{Yes} \\
5 \mathrm{No}\end{array}$ \\
\hline Mean \pm SD & & $58 \pm 16$ & $466 \pm 1028$ & & & $1.2 \pm 0.9$ & $8.3 \pm 2.7$ & \\
\hline Median & & 64 & 65 & & & 1 & 9 & \\
\hline
\end{tabular}

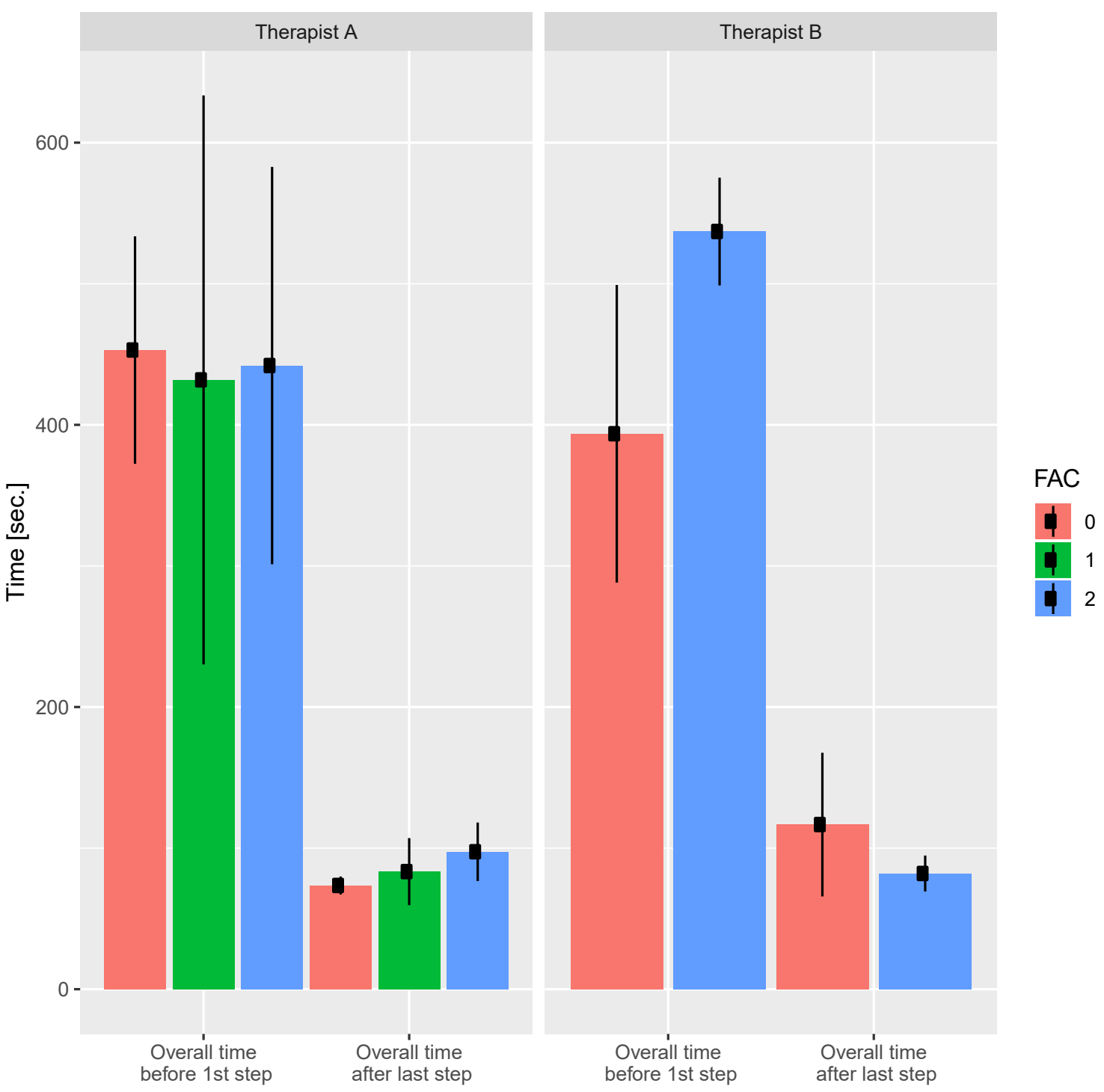

Figure 1. Overall times needed before and after training on the Andago 
After individual definition of the optimum setup, all patients had a BWS between 5-20 kg on each side, a medium fall limit $(12 \mathrm{~cm})$ and in $73 \%$ of the cases a slow maximum speed $(0.8 \mathrm{~km} / \mathrm{h})$. Turning offset in order to counteract gait deviation was consistently used only with one patient. During the training session the average walking distance was $48 \pm 29 \mathrm{~m}$ in the PFM, $51 \pm 30 \mathrm{~m}$ in the SLM and $71 \pm 48 \mathrm{~m}$ in the MAM. Three patients (ID: 2, 7 and 10) terminated the training session prematurely after 80,11 and $43 \mathrm{~m}$ (FAC: $0 / 0 / 1$ ) due to fatigue. During the therapy session, the average walking distance was $91 \pm 35$ meters and the average walking time was $798 \pm 370 \mathrm{sec}$. The same three patients mentioned before terminated the therapy session prematurely after 72 , 2 and $30 \mathrm{~m}$. We descriptively observed longer achieved distances during both sessions in patients with a less severe FAC: training session FAC 0 vs. $2, p<0.0001$, FAC 1 vs. $2, p<0.01$; therapy session $\mathrm{p}$ not calculated due to the predefined maximum distance (Figure 2).

Near-falls and emergency stops did not occur during any of the sessions. Therapist interventions consisting of correcting the direction or switching to the manual mode were more frequent during the training sessions. Collisions $(n=58)$ were only observed during the therapy sessions, which were performed in the PFM unless the therapist had to intervene. Both observations are explained by the experimental setup with the task to follow a straight line and frequent turns in the semicircle during the training sessions and the presence of obstacles only in the therapy session, respectively. A higher number of verbal instructions and stops by the therapist was also necessary during the therapy sessions (Table 2).

Overall satisfaction with the Andago therapy is shown in more detail in figure $3.85 \%$ of the patients were satisfied to very satisfied with the therapy. Only one patient (ID 10), who was hardly able to use the device, reported dissatisfaction. The therapists were in equal amounts either satisfied (46\%) or reported to be neither satisfied nor dissatisfied (46\%).

In comparison to a gait training on the Lokomat, a slight majority of the patients favoured Andago over Lokomat (Table 3, item 10) independently from their gait abilities represented by the FAC. Therapists favoured in general the Lokomat, however not in patients with a FAC $\geq 1$ (Figure 4).

Table 2. ID, type and number of events (n) during the training and therapy sessions

\begin{tabular}{|c|c|c|c|c|}
\hline ID & Event & Description / Type & Training session (n) & Therapy session (n) \\
\hline 1 & Near-fall & Patient stumbles and would have fallen if not held by the Andago harness & 0 & 0 \\
\hline 2 & Collision & Pat. collides with an object in the environment & 0 & 29 (17 left, 17 right) \# \\
\hline 3 & Collision and stop & $\begin{array}{l}\text { Pat. collides with an object in the environment prompting the therapist to stop the } \\
\text { device }\end{array}$ & 0 & 29 (28 left, 3 right) $\#$ \\
\hline 4 & Intervention by therapist & Therapist stops the device in order to impede a collision or re-adjust the device & 5 & 56 \\
\hline 5 & Intervention by therapist & Therapist takes control by changing into MAM & 53 & 23 \\
\hline 6 & Intervention by therapist & Therapist corrects the direction & 52 & 13 \\
\hline 7 & Intervention by therapist & Verbal instruction by the therapist & 33 & 102 \\
\hline 8 & Emergency stop & The Andago stops due to the usage of the emergency button & 0 & 0 \\
\hline 9 & Error message & The Andago shows an error message & 2 & 9 \\
\hline 10 & Break & $\begin{array}{l}\text { The patient requests a pause, or the session is briefly interrupted for other reasons (e.g. } \\
\text { letting other people passing by) }\end{array}$ & 0 & 3 \\
\hline 11 & Physical contact with the device & Types of physical contact: 3 & 39 & 28 \\
\hline 12 & (Serious) AE / ADE & According to ISO 14155:2011 (www.iso.org) & 0 & 0 \\
\hline \multirow[t]{2}{*}{13} & Device deficiency & Types of device deficiencies: 4 & 16 & 5 \\
\hline & Total number & & 200 & 297 \\
\hline
\end{tabular}

\# sometimes simultaneous collisions on both sides

Table 3. Patient and therapist satisfaction with the Andago

\begin{tabular}{|c|c|c|c|c|c|c|c|c|c|c|c|c|}
\hline \multirow{3}{*}{ Item } & \multicolumn{6}{|c|}{ Training session } & \multicolumn{6}{|c|}{ Therapy session } \\
\hline & \multicolumn{3}{|c|}{ Patient } & \multicolumn{3}{|c|}{ Therapist } & \multicolumn{3}{|c|}{ Patient } & \multicolumn{3}{|c|}{ Therapist } \\
\hline & yes & no & n.a. & yes & no & n.a. & yes & no & n.a. & yes & no & n.a. \\
\hline 1. Difficulty putting the harness on & $0 \%$ & $100 \%$ & $0 \%$ & $0 \%$ & $100 \%$ & $0 \%$ & $0 \%$ & $100 \%$ & $0 \%$ & $0 \%$ & $100 \%$ & $0 \%$ \\
\hline $\begin{array}{l}\text { 2. Difficulty attaching the harness to the frame and lifting } \\
\text { the patient }\end{array}$ & $0 \%$ & $100 \%$ & $0 \%$ & $8 \%$ & $92 \%$ & $0 \%$ & $0 \%$ & $100 \%$ & $0 \%$ & $8 \%$ & $92 \%$ & $0 \%$ \\
\hline 3. Perception of asymmetrical BWS as useful & $15 \%$ & $15 \%$ & $70 \%$ & $31 \%$ & $8 \%$ & $61 \%$ & $15 \%$ & $15 \%$ & $70 \%$ & $15 \%$ & $23 \%$ & $62 \%$ \\
\hline $\begin{array}{l}\text { 4a. Feeling of sufficient protection against falls by setting } \\
\text { the fall limit }\end{array}$ & $100 \%$ & $0 \%$ & $0 \%$ & - & - & - & $100 \%$ & $0 \%$ & $0 \%$ & - & - & - \\
\hline 4b. Difficulty adjusting the fall limit & - & - & - & $8 \%$ & $92 \%$ & $0 \%$ & - & - & - & $0 \%$ & $100 \%$ & $0 \%$ \\
\hline 5a. Satisfaction with speed & $100 \%$ & $0 \%$ & $0 \%$ & - & - & - & $77 \%$ & $23 \%$ & $0 \%$ & - & - & - \\
\hline 5b. Difficulty adjusting speed & - & - & - & $0 \%$ & $100 \%$ & $0 \%$ & - & - & - & $0 \%$ & $100 \%$ & $0 \%$ \\
\hline 6. Usefulness of turning offset & - & - & - & $15 \%$ & $0 \%$ & $85 \%$ & - & - & - & - & - & - \\
\hline 7a. Satisfaction with PFM / user-friendliness of PFM & $84 \%$ & $16 \%$ & $0 \%$ & $46 \%$ & $54 \%$ & $0 \%$ & - & - & - & - & - & - \\
\hline 7b. Satisfaction with SLM / user-friendliness of SLM & $92 \%$ & $0 \%$ & $8 \%$ & $31 \%$ & $69 \%$ & $0 \%$ & - & - & - & - & - & - \\
\hline 7c. Satisfaction with MAM / user-friendliness of MAM & $84 \%$ & $8 \%$ & $8 \%$ & $62 \%$ & $38 \%$ & $0 \%$ & - & - & - & - & - & - \\
\hline $\begin{array}{l}\text { 8. Suitability of Andago for } 90^{\circ} \text { - or } 180^{\circ} \text {-turns and } \\
\text { passages }\end{array}$ & - & - & - & - & - & - & $77 \%$ & $23 \%$ & $0 \%$ & $54 \%$ & $46 \%$ & $0 \%$ \\
\hline 9. Difficulty dismounting the patient from Andago & $8 \%$ & $92 \%$ & $0 \%$ & $0 \%$ & $100 \%$ & $0 \%$ & $0 \%$ & $100 \%$ & $0 \%$ & $0 \%$ & $100 \%$ & $0 \%$ \\
\hline 10. Preference of Andago over Lokomat ${ }^{\circledR}$ therapy & - & - & - & - & - & - & $54 \%$ & $8 \%$ & $38 \%$ & $38 \%$ & $62 \%$ & $0 \%$ \\
\hline 11. Overall satisfaction with Andago & - & - & - & - & - & - & $92 \%$ & $8 \%$ & $0 \%$ & $92 \%$ & $8 \%$ & $0 \%$ \\
\hline
\end{tabular}




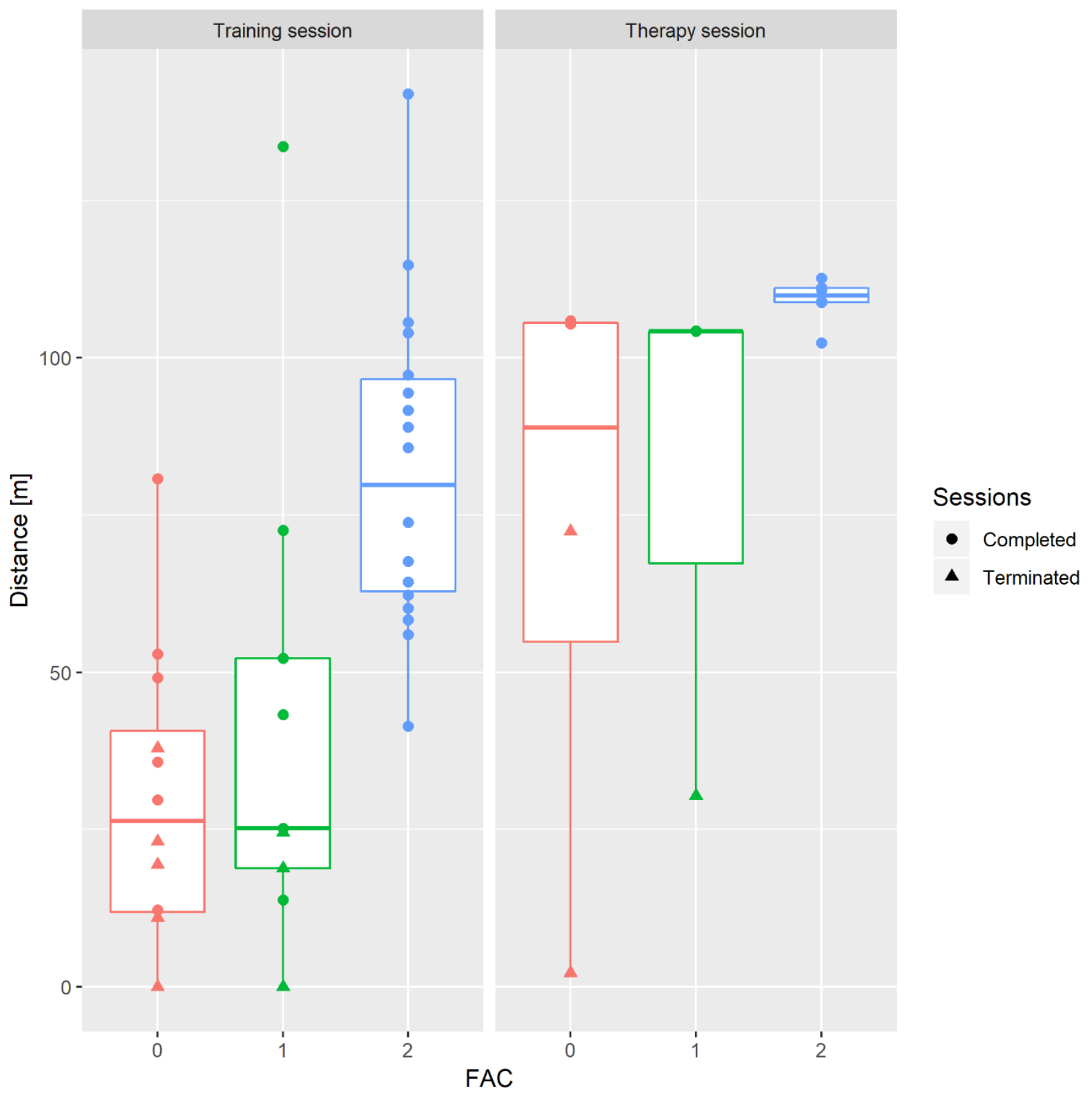

Figure 2. Achieved distances during the training and therapy sessions
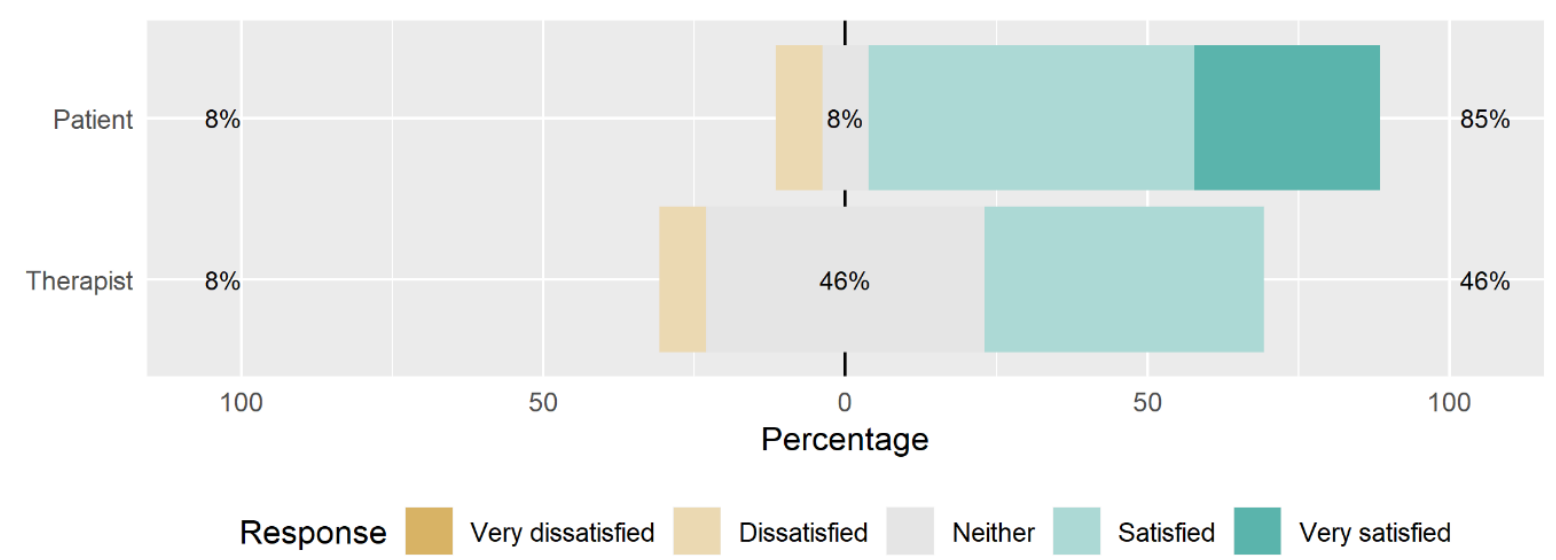

Figure 3. Overall satisfaction with the Andago V2.0 


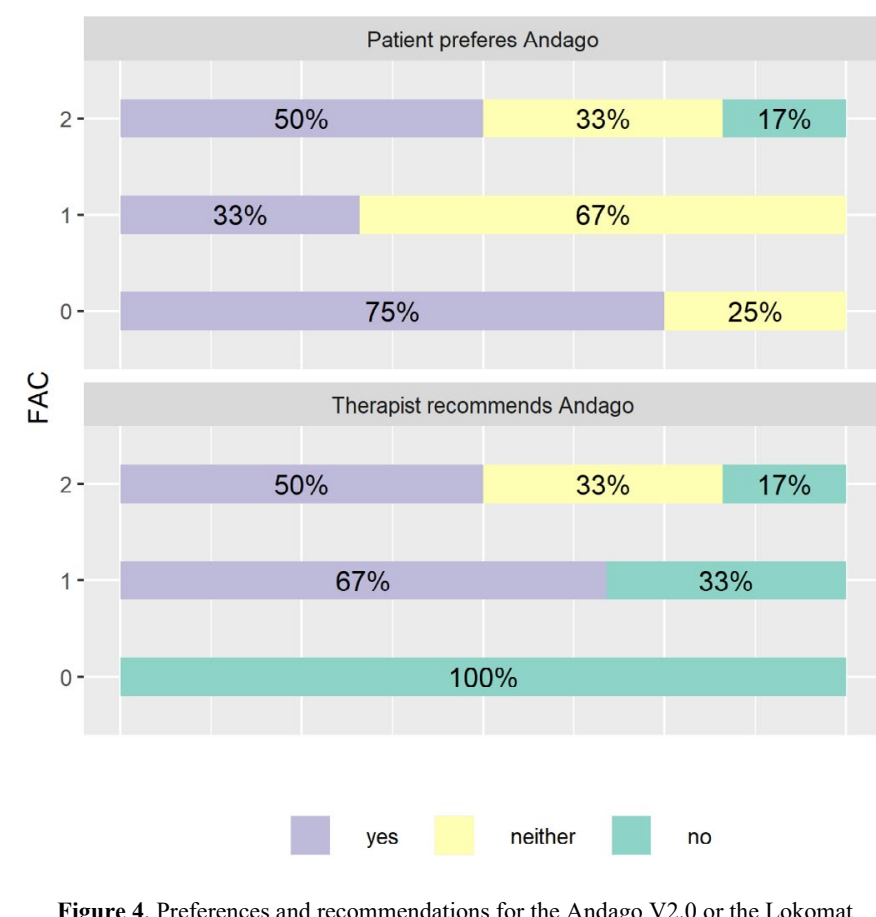

Figure 4. Preferences and recommendations for the Andago V2.0 or the Lokomat

\section{0-MWT}

At the time of the therapy session five participants were able to perform the 10-MWT under the supervision of a therapist $(n=1)$ or with the use of a cane or a walking frame $(n=4)$. With the Andago eleven patients were able to perform the 10-MWT $(p=0.001)$.

\section{Safety}

The overall number of AE, SAE, ADE or device deficiencies is also reported in table 2. AE, SAE or ADE did not occur. Despite the use of a prototype, only a few minor device deficiencies were observed. The therapists were not able to set the fall limit adequately 15 times in five patients, usually lifting the patient slightly from the ground. According to the personal experience of the authors, this device deficiency has not been observed in the CE-certified version. This deficiency was only partially reflected by the answers to item 2 in the patient and therapist questionnaires.

Three times the remote control did not work, twice the brakes were not released and once Andago had to be rebooted. Three error messages referred to a detected stumbling. These error messages occurred, because the patients let themselves sink into the harness, but the therapists did not report these events as near-falls. Eight error messages indicated that the left suspension rail had to be lowered.

All sessions could be performed safely, although therapists had to intervene, e.g., to avoid collisions. We frequently observed a physical contact of the patient with the Andago. Typically, patients touched the frame with the paretic foot. We also observed that the space between the hands and the obstacles in narrow passages like door frames (min. width $90 \mathrm{~cm}$ ) was sometimes tight. One patient touched the Andago frame with his head. Our therapists therefore paid particular attention to collisions with obstacles or physical contact to Andago to minimize any hypothetical risk of injury. In a post-hoc analysis patients with concomitant hemianopia and/or neglect featured more frequently collisions, interventions by the therapist and physical contacts with the device during the therapy session $(p=0.03)$, but not during the training session (no significant difference) (Figure 5).

\section{Discussion \\ Usability and acceptance}

This is to our knowledge the first usability study with the Andago V2.0 prototype. The aim of the present study was to address its usability and acceptance in adult stroke patients in a neurorehabilitation clinic. All interventions were safe. All patients were able to perform the Andago training except for one patient with a FAC of 0 , who was hardly able to move. Usability of the Andago as measured by the preparation and follow-up times seems adequate for everyday routine: up to 10 minutes must be planned for the set-up and release of the patient. The usability of the Andago was also supported by the results of the 10-MWT. Significantly more patients were able to perform the 10MWT using the Andago than patients with a cane or a walker. This observation suggests that an advantage of the Andago consists of its usage in patients who still need physical support in order to ambulate. The Andago was in general well accepted by patients and therapists. Most patients and therapists in approximately half the cases were either very satisfied or satisfied with this therapy. Dissatisfaction by patients or therapists was reported only in $8 \%$ of all cases. The other results of the satisfaction questionnaires also indicated a high acceptance of the Andago. Importantly, all patients felt sufficiently protected from falls while using the Andago. Therapists apparently preferred the manual mode to the patient-following and straight-line mode, which may reflect the higher degree of control provided by the manual mode. Besides asymmetrical BWS and turning offset were rarely used in this small usability study.

Altogether more patients preferred the Andago over the Lokomat therapy, which may be due to the subjective experience of "real walking". Therapists preferred the Andago therapy only in patients with less severe gait disorder. Patients with a moderate gait disorder as defined by a FAC

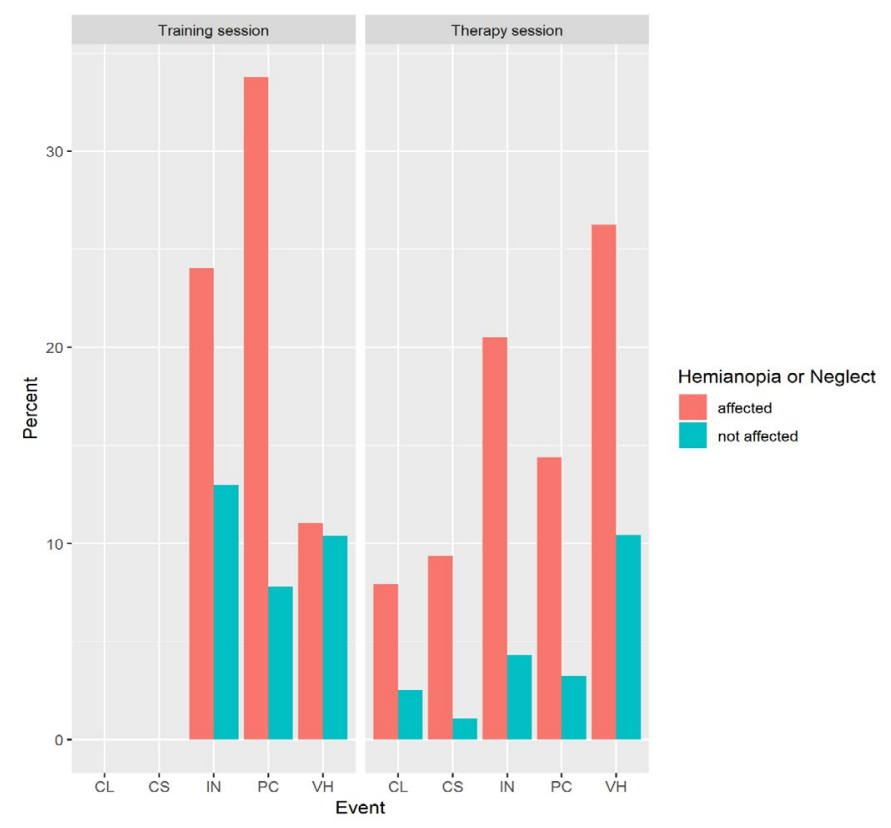

Figure 5. Percentage of events in patients with visuospatial impairment $\mathrm{CL}$ - collision, $\mathrm{CS}$ - collision with stop, IN - intervention, $\mathrm{PC}$ - physical contact, $\mathrm{VH}$ verbal help 
of 2 also achieved greater distances with the Andago than patients with a severe gait disorder. Considering these data, it can be assumed, that the Andago is useful for patients who are already able to initiate steps, but show difficulty wearing body weight and performing balance tasks. Such candidates for Andago therapy may usually feature a FAC of 2 (or higher). The Andago can also be used in patients featuring a FAC of 0 -1, although therapists clearly preferred Lokomat therapy in patients with a FAC of 0 .

Studies using a larger sample of stroke patients and including a control group should investigate under which circumstances Andago may be used as a supplement to - or possibly as a replacement of robot-assisted gait devices. The add-on value of Andago is apparently the possibility of walking overground, i.e., being a step closer to walking in daily life. The BWS offers the possibility to dose the intensity of the training progressively according to the patient's capabilities. In addition, the harness gives patients the feeling of safety allowing them to focus on walking longer distances. However, we noticed that patients sometimes overestimated their motor skills to use the device properly, hypothetically due to anosognosia or an incorrect appraisal of their gait disorder.

\section{Safety}

All training sessions could be performed safely. AE or ADE did not occur. Only a few minor device deficiencies or error messages were recorded. However, we observed a considerable number of collisions or near-collisions with obstacles and physical contacts of the patient with the device especially during the therapy session. The therapists had to intervene with the remote control or give verbal instructions quite frequently, e. g., when the Andago had to be steered accurately to avoid collisions when passing through doors or turning 90 or $180^{\circ}$. The therapists rated the Andago with a $54 \%$ suitability in these situations.

We therefore performed a subgroup analysis with patients suffering from hemianopia and/or neglect and found that these patients featured a significantly higher number of collisions, interventions by the therapists and physical contacts with the device than the subgroup without a clinically relevant visuospatial impairment. These data suggest that candidates for an Andago therapy should be screened for visual field disorders and/ or neglect. We recommend paying attention to such patients during the sessions in order to minimize a hypothetical injury risk.

A limitation of this study is that our selection of patients was not normally distributed. The inclusion of only two patients with a lefthemispheric stroke might have biased our results due to the higher prevalence of neglect in eight of the remaining eleven patients with a right-hemispheric stroke [15-18]. Therefore, our results may not be representative for stroke patients in general.

Interestingly, two patients with long-term chronic stroke ( $>3$ years) participated in this study indicating that the Andago may not only be useful in patients with subacute stroke, but also in patients with chronic stroke. Future studies should address the efficacy of the Andago therapy with patients in different stages of haemorrhagic or ischemic stroke and in other neurological diseases such as multiple sclerosis, Parkinson`s disease or Guillain-Barré syndrome.

\section{Implications for rehabilitation}

- The Andago seems to be a valuable device for overground mobility training in patients presenting moderate to severe gait disorders after stroke.

- Short set-up times and an overall high satisfaction by patients and therapists make it a promising adjunct to robot-assisted as well as conventional overground gait therapy.

\section{Acknowledgment}

We thank all patients and staff who participated in this study.

Rehaklinik Zihlschlacht is a Clinical Partner of HOCOMA. The Andago V2.0 prototype was provided free of charge for the performance of this study.

\section{Disclosure of interest}

The authors declare no conflict of interest. All authors disclosed any financial and personal relationships with other people or organizations that could inappropriately influence (bias) their work.

\section{References}

1. Giladi N, Horak FB, Hausdorff JM (2013) Classification of gait disturbances: distinguishing between continuous and episodic changes. Mov Disord 28: 1469-1473. [Crossref]

2. Balaban B, Tok F (2014) Gait disturbances in patients with stroke. PM R 6: 635-642. [Crossref]

3. Dundar U, Toktas H, Solak O, Ulasli AM, Eroglu S (2014) A comparative study of conventional physiotherapy versus robotic training combined with physiotherapy in patients with stroke. Top Stroke Rehabil 21: 453-461. [Crossref]

4. Mehrholz J, Elsner B, Werner C, Kugler J, Pohl M, et al. (2013) Electromechanical-assisted training for walking after stroke. Cochrane Database Syst Rev 5: CD006185.[Crossref]

5. Richards CL, Malouin F, Wood-Dauphinee S, Williams JI, Bouchard JP, et al. (1993) Task-specific physical therapy for optimization of gait recovery in acute stroke patients. Arch Phys Med Rehabil 74: 612-620. [Crossref]

6. Alton F, Baldey L, Caplan S, Morrissey MC (1998) A kinematic comparison of ove ground and treadmill walking. Clin Biomech (Bristol, Avon) 13: 434-440. [Crossref]

7. Kwakkel G, van Peppen R, Wagenaar RC, Wood Dauphinee S, Richards C, et al. (2004) Effects of augmented exercise therapy time after stroke: a meta-analysis. Stroke. 35: 2529-2539. [Crossref]

8. Mayr A, Kofler M, Quirbach E, Matzak H, Fröhlich K, et al. (2007) Prospective, blinded, randomized crossover study of gait rehabilitation in stroke patients using the Lokomat gait orthosis. Neurorehabil Neural Repair 21: 307-314. [Crossref]

9. Jezernik S, Colombo G, Keller T, Frueh H, Morari M (2003) Robotic orthosis lokomat a rehabilitation and research tool. Neuromodulation 6: 108-115. [Crossref]

10. Holden MK, Gill KM, Magliozzi MR, Nathan J, Piehl-Baker L (1984) Clinical gai assessment in the neurologically impaired. Reliability and meaningfulness. Phys Ther 64: 35-40. [Crossref]

11. Criddle LM, Bonnono C, Fisher SK (2003) Standardizing stroke assessment using the National Institutes of Health Stroke Scale. J Emerg Nurs 29: 541-546. [Crossref]

12. Adams HP, Bendixen BH, Kappelle LJ, Biller J, Love BB, et al. (1993) Classification of subtype of acute ischemic stroke. Definitions for use in a multicentre clinical trial. TOAST. Trial of Org 10172 in Acute Stroke Treatment. Stroke 24: 35-41. [Crossref]

13. Kwah LK, Diong J (2014) National Institutes of Health Stroke Scale (NIHSS). J Physiother 60: 61. [Crossref]

14. Bohannon RW (1997) Comfortable and maximum walking speed of adults aged 20-79 years: reference values and determinants. Age Ageing 26: 15-19. [Crossref]

15. Denes G, Semenza C, Stoppa E, Lis A (1982) Unilateral spatial neglect and recovery from hemiplegia: a follow-up study. Brain 105: 543-552. [Crossref]

16. Katz N, Hartman-Maeir A, Ring H, Soroker N (1999) Functional disability and rehabilitation outcome in right hemisphere damaged patients with and without unilateral spatial neglect. Arch Phys Med Rehabil 80: 379-384. [Crossref]

17. Paolucci S, Antonucci G, Grasso MG, Pizzamiglio L (2001) The role of unilatera spatial neglect in rehabilitation of right brain-damaged ischemic stroke patients: a matched comparison. Arch Phys Med Rehabil 82: 743-749. [Crossref]

18. Gottesman RF, Kleinman JT, Davis C, Heidler-Gary J, Newhart M, et al. (2008) Unilateral neglect is more severe and common in older patients with right hemispheric stroke. Neurology 71: 1439-1444. [Crossref]

Copyright: (C2019 Marks D. This is an open-access article distributed under the terms of the Creative Commons Attribution License, which permits unrestricted use, distribution, and reproduction in any medium, provided the original author and source are credited. 